\title{
BACTERIAL UPTAKE OF HABEKACIN, A NOVEL AMINOGLYCOSIDE ANTIBIOTIC
}

\author{
Keita Matsunaga, Toshio Nishimura and Nobuo Tanaka \\ Institute of Applied Microbiology, University of Tokyo, \\ Bunkyoku, Tokyo 113, Japan
}

(Received for publication October 4, 1983)

\begin{abstract}
Cellular uptake of habekacin, 1-N-(4-amino-2-hydroxybutyryl)dibekacin, was studied by incubating exponentially growing culture of Escherichia coli Q13 and its kanamycin-resistant mutants with $\left[{ }^{3} \mathrm{H}\right]$ habekacin. Kanamycin-resistant mutants, in which the resistance is due to alteration of ribosomes, were cross-resistant to habekacin, and showed a lower uptake of $\left[{ }^{3} \mathrm{H}\right]$ habekacin than the parental cells, suggesting that binding to ribosomes accelerates cellular uptake of habekacin. Cellular accumulation of $\left[{ }^{3} \mathrm{H}\right]$ habekacin by wild type cells was markedly inhibited by low temperature and by 2,4-dinitrophenol, suggesting that uptake of habekacin involves energy-dependent transport. The uptake of $\left[{ }^{3} \mathrm{H}\right]$ habekacin was reduced by various aminoglycoside antibiotics, suggesting common transport systems and/or common internal binding sites on the ribosome. Intracellular accumulation of $\left[{ }^{3} \mathrm{H}\right]$ dibekacin was reduced by habekacin, suggesting that both antibiotics possess a common transport system and/or common binding sites on the ribosome. Dibekacin was a better competitor than amikacin, suggesting that the dibekacin moiety of habekacin molecule, but not the 4-amino-2-hydroxybutyryl moiety, participates in the transport and/or binding to the ribosome. Binding of $\left[{ }^{3} \mathrm{H}\right]$ habekacin to $E$. coli ribosomes was reversed by various aminoglycosides and the degree of inhibition paralleled the one of cellular uptake, suggesting that competition by aminoglycosides for the habekacin uptake occurs at the ribosomal level.
\end{abstract}

Although bacterial uptake of aminoglycoside antibiotics has been extensively investigated ${ }^{1 \sim 8)}$, the mechanism of uptake still remains to be determined. Kinetic studies have shown three sequential phases of cellular uptake: (1) an initial energy-independent ionic binding to the cell surface, (2) EDP-I (energydependent phase I), a slow rate of uptake, and (3) EDP-II, an enhanced rate of uptake, which is initiated by binding of aminoglycoside antibiotics to ribosomes ${ }^{3 \sim 6)}$. The initial energy-independent process involves diffusion across the outer membrane of Gram-negative bacteria through the pores formed by porin proteins ${ }^{14)}$.

Habekacin, 1- $N$-[(S)-4-amino-2-hydroxybutyryl]dibekacin, is a novel amino acid-containing aminoglycoside antibiotic, with a broad antibacterial spectrum, including Pseudomonas aeruginosa, and some kanamycin-, gentamicin-, and tobramycin-resistant organisms ${ }^{12,13)}$. Clinical investigations on habekacin are now in progress.

We have studied the mechanism of action of habekacin ${ }^{7)}$. Electron microscopic examination has revealed that "blebs", derived from the outer membrane, are formed on cells treated with habekacin. Cytoplasmic contents leak into the lumina of blebs, and the membrane of some enlarged blebs is disrupted. In a cell-free system, habekacin binds to both $50 \mathrm{~S}$ and $30 \mathrm{~S}$ ribosomal subunits, interferes with protein synthesis, induces codon misreading, and inhibits the translocation process.

Since the cellular uptake of amino acid-containing aminoglycoside antibiotics, such as amikacin, fortimicin, and related substances, has been not well investigated, we have studied bacterial uptake of habekacin, and the results are presented in this publication. 


\section{Materials and Methods}

Habekacin (Fig. 1), dibekacin, $\left[{ }^{3} \mathrm{H}\right]$ habekacin $(44.4 \mathrm{mCi} / \mathrm{mmol})$, and $\left[{ }^{3} \mathrm{H}\right]$ dibekacin $(47.2 \mathrm{mCi} /$ $\mathrm{mmol}$ ) were generously provided by Meiji Seika Kaisha, Ltd., Tokyo; and derivatives of habekacin, shown in Table 1, by Dr. H. Umezawa, Institute of Microbial Chemistry, Tokyo ${ }^{10,11}$.

Kanamycin-resistant mutants were isolated after treating Escherichia coli Q13 with $N$-methyl- $N^{\prime}$ nitro- $N$-nitrosoguanidine ${ }^{8,8)}$. The resistance of R1-4, R2-1 and R3-3 mutants was attributed to alterations of the $30 \mathrm{~S}$ ribosomal subunit, and that of R3-5 to a change in the 50S subunit.

Cellular uptake or accumulation of antibiotics was carried out by incubating $\left[{ }^{3} \mathrm{H}\right]$ habekacin or $\left[{ }^{3} \mathrm{H}\right]-$ dibekacin with $E$. coli cells in the logarithmic phase of growth. The cells were collected on Millipore filters and washed with phosphate-buffered saline, and radioactivity was assayed in a scintillation counter.

The binding of $\left[{ }^{3} \mathrm{H}\right]$ habekacin to $E$. coli ribosomes was determined by equilibrium dialysis as described previously ${ }^{15)}$.

\section{Results}

Uptake of $\left[{ }^{3} \mathrm{H}\right]$ Habekacin by Sensitive and Resistant Cells of E. coli

A culture of $E$. coli Q13 growing exponentially in nutrient broth was treated at zero time with $\left[{ }^{3} \mathrm{H}\right]$ habekacin $(2 \mu \mathrm{g} / \mathrm{ml})$. The cells accumulated the antibiotic, and the kinetics of habekacin uptake were linear for 30 minutes with the initial binding followed by a lag period of several minutes (Fig. 2). In a similar experiment, uptake of habekacin occurred in the kanamycin-resistant mutants, R1-4, R2-1,

Fig. 1. Structure of habekacin.

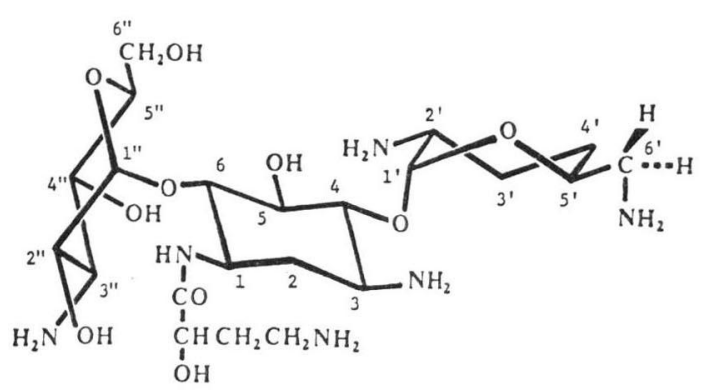

Table 1. The sensitivity of E. coli Q13 and its kanamycin-resistant mutants to kanamycin and habekacin.

\begin{tabular}{lcc}
\hline \multirow{2}{*}{ Strain } & \multicolumn{2}{c}{$\begin{array}{c}\text { Minimal inhibitory } \\
\text { concentration }(\mu \mathrm{g} / \mathrm{ml})\end{array}$} \\
\cline { 2 - 3 } & Kanamycin & Habekacin \\
\hline Parent & 3.1 & 1.6 \\
R1-4 & 100 & 25 \\
R2-1 & 50 & 25 \\
R3-3 & 100 & 25 \\
R3-5 & 100 & 25 \\
\hline
\end{tabular}

The minimal inhibitory concentration was determined by agar streak method. The medium used was heart infusion agar (Difco).

Habekacin $1 \mu \mathrm{g} / \mathrm{ml}=1.81 \mu \mathrm{M}$.
Fig. 2. Cellular uptake of $\left[{ }^{3} \mathrm{H}\right]$ habekacin by $E$. coli Q13 and its kanamycin-resistant mutants.

$\left[{ }^{3} \mathrm{H}\right]$ Habekacin $(2 \mu \mathrm{g} / \mathrm{ml})$ was added to an exponentially growing culture of $E$. coli in nutrient broth at zero time.

E. coli Q13 (A). Kanamycin-resistant mutants:

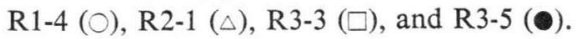

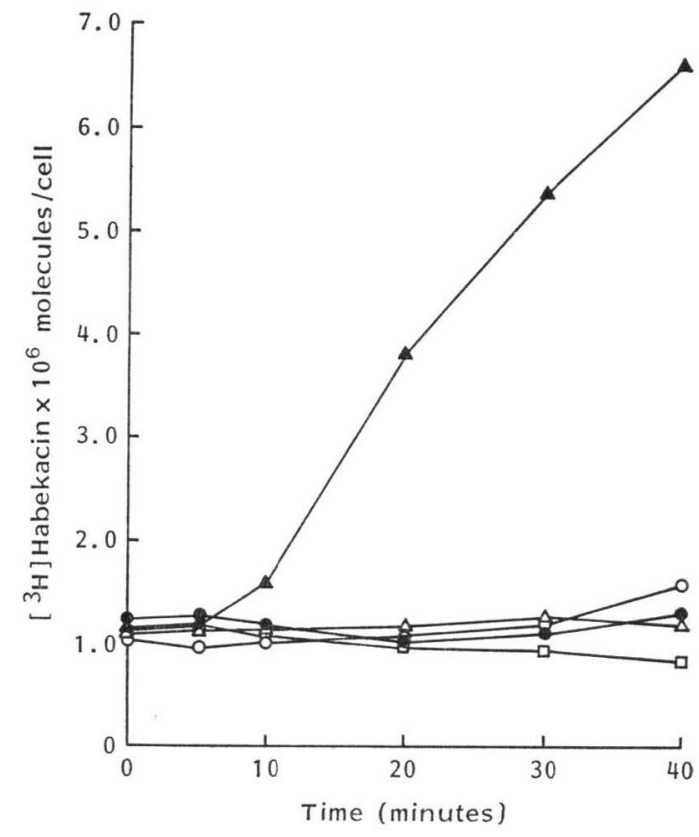


R3-3, and R3-5, to a much lower extent than in the sensitive parent strain (Fig. 2). All the resistant mutants, in which the resistance is attributed to alteration of the ribosomes ${ }^{8,8)}$, displayed cross-resistance to habekacin (Table 1). These results suggest that the binding to ribosomes accelerates cellular uptake of habekacin.

\section{Effects of 2,4-Dinitrophenol and Low Temperature on $\left[{ }^{3} \mathrm{H}\right]$ Habekacin Uptake}

Cellular accumulation of $\left[{ }^{3} \mathrm{H}\right]$ habekacin by $E$. coli Q13 was markedly inhibited by low temperature and by 2,4-dinitrophenol ( $2 \mathrm{~mm}$ ) added at zero time or 15 minutes after the initial exposure to habekacin (Fig. 3). These results suggest that bacterial uptake of habekacin involves energy-dependent transport system.

Effects of Various Aminoglycoside Antibiotics and Their Components

\section{on Uptake of $\left[{ }^{3} \mathrm{H}\right]$ Habekacin}

The accumulation of $\left[{ }^{3} \mathrm{H}\right]$ habekacin $(2 \mu \mathrm{M})$ in sensitive cells was reduced by the addition of dibekacin, and amikacin showed less effect than dibekacin. The results of kinetic studies, in comparison with the effect of unlabelled habekacin, are illustrated in Fig. 4.

Fig. 3. Inhibition of $\left[{ }^{3} \mathrm{H}\right]$ habekacin accumulation in E. coli Q13 cells by 2,4-dinitrophenol and low temperature.

$\left[{ }^{3} \mathrm{H}\right]$ Habekacin $(2 \mu \mathrm{g} / \mathrm{ml})$ was added at zero time to an exponentially growing culture of $E$. coli in nutrient broth.

Control $(\square)$. 2,4-Dinitrophenol was added to the mixture at zero time (-) or at 15 minutes $(0)$. The culture was transferred to ice bath at zero time (0) or at 15 minutes $(\triangle)$.

Both procedures, introduced at zero time, resulted in the same degree of inhibition.

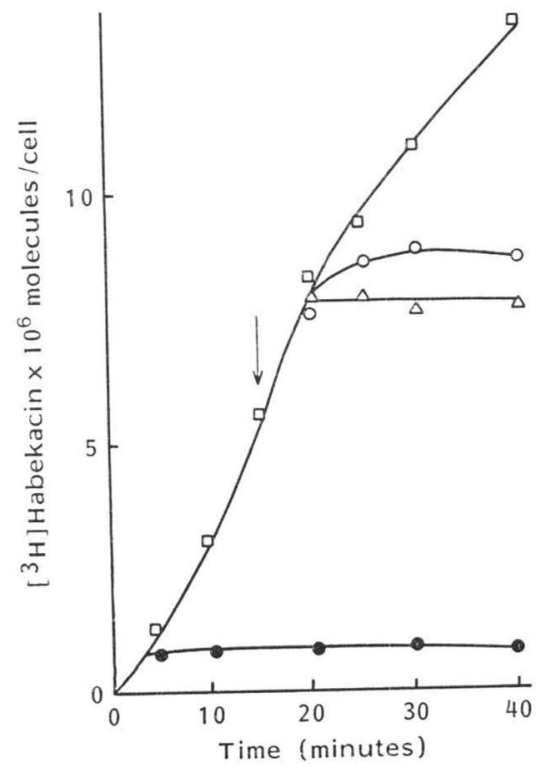

Fig. 4. Kinetics of uptake of $\left[{ }^{3} \mathrm{H}\right]$ habekacin by $E$. coli cells in the presence or absence of amikacin, habekacin or dibekacin.

Cells of E. coli Q13, at the logarithmic phase of growth, were incubated at $37^{\circ} \mathrm{C}$ with $2 \mu \mathrm{M}\left[{ }^{3} \mathrm{H}\right]-$ habekacin in the presence of $10 \mu \mathrm{M}$ amikacin $(\triangle)$, $10 \mu \mathrm{M}$ habekacin (๑) or $10 \mu \mathrm{M}$ dibekacin (O). Control ( $\square)$.

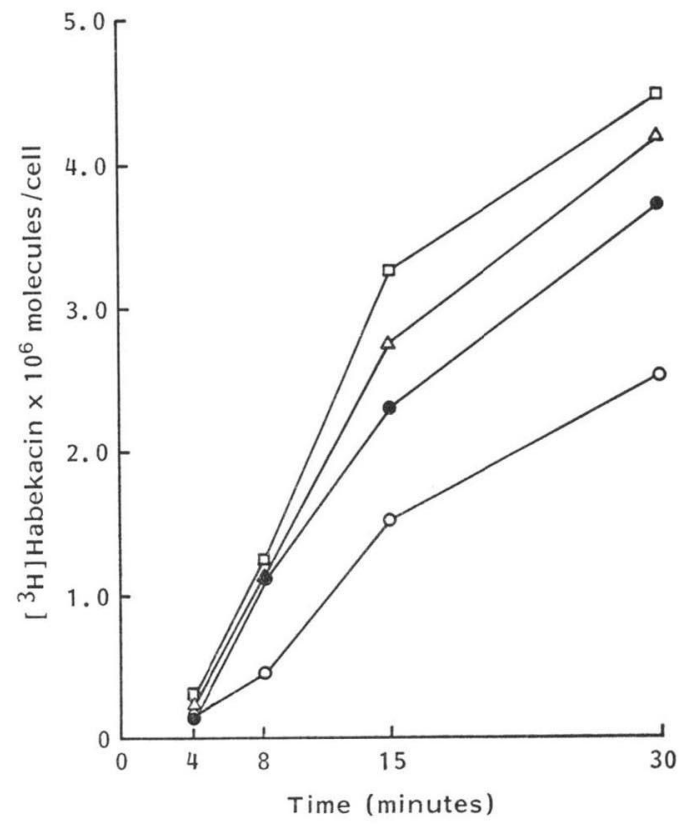


Table 2. Relative uptake of $\left[{ }^{3} \mathrm{H}\right]$ habekacin by $E$. coli cells in the presence of various aminoglycoside antibiotics and their components.

\begin{tabular}{lcc|lcc}
\hline \multicolumn{1}{c}{ Addition } & $+2 \mu \mathrm{M}$ & $+10 \mu \mathrm{M}$ & \multicolumn{1}{c}{ Addition } & $+2 \mu \mathrm{M}$ & $+10 \mu \mathrm{M}$ \\
\hline Habekacin (HBK) & 86 & 63 & $6^{\prime \prime}$-Deoxy-HBK & 87 & 78 \\
Dibekacin & 79 & 44 & $4^{\prime \prime}, 6^{\prime \prime}$-Dideoxy-HBK & 91 & 77 \\
Amikacin & 98 & 86 & 5-Deoxy-HBK & 85 & 67 \\
Kanamycin & 93 & 85 & 5-Deoxy-6'- -methyl-HBK & 89 & 77 \\
Streptomycin & 81 & 76 & 5,6"-Dideoxy-HBK & 84 & 51 \\
Neamine & 91 & 87 & 5,4",6"-Trideoxy-HBK & 97 & 73 \\
Ribostamycin & 92 & 83 & 4-Amino-2-hydroxybutyric & 101 & 108 \\
Neomycin & 72 & 29 & acid & 110 & 101 \\
Kasugamycin & 98 & 93 & 3-Amino-3-deoxyglucose & 110 & 102 \\
Dactimicin & 94 & 81 & 2-Deoxystreptamine & 102 & 115 \\
Fortimicin A & 98 & 81 & Glucose & [ ${ }^{3}$ H]Habekacin & 171 \\
\end{tabular}

The number represents relative uptake of $\left[{ }^{3} \mathrm{H}\right]$ habekacin, and $100 \%$ uptake is $3.7 \times 10^{6}$ habekacin molecules/cell. The exponentially growing cells of $E$. coli Q13 were incubated with $2 \mu \mathrm{M}\left[{ }^{3} \mathrm{H}\right]$ habekacin at $37^{\circ} \mathrm{C}$ for 15 minutes in the presence or absence of $2 \mu \mathrm{M}$ or $10 \mu \mathrm{M}$ antibiotic or its components, and transferred to ice bath.

The bacterial uptake of $\left[{ }^{3} \mathrm{H}\right]$ habekacin was also decreased in the presence of kanamycin, streptomycin, neamine, ribostamycin, neomycin, dactimicin, fortimicin A, 6"-deoxyhabekacin, 4",6" -dideoxyhabekacin, 5-deoxyhabekacin, 5-deoxy- $6^{\prime}-N$-methylhabekacin, 5,6"-dideoxyhabekacin, or 5,4" $6^{\prime \prime}$ trideoxyhabekacin; but not by 4-amino-2-hydroxybutyric acid, 3-amino-3-deoxyglucose, 2-deoxystreptamine or glucose. Kasugamycin showed a marginal effect (Table 2). The results suggest that either the transport system for habekacin or the internal binding site, presumably the ribosome, is common to these antibiotics. Glucose addition increased the uptake of $\left[{ }^{3} \mathrm{H}\right]$ habekacin, probably because of energy supply.

\section{Uptake of $\left[{ }^{3} \mathrm{H}\right]$ Dibekacin by $E$. coli Q13}

A culture of $E$. coli Q13 in the logarithmic phase of growth was incubated with $\left[{ }^{3} \mathrm{H}\right]$ dibekacin $(2 \mu \mathrm{M})$ at $37^{\circ} \mathrm{C}$ for 15 minutes. The cells accumulated $\left[{ }^{3} \mathrm{H}\right]$ dibekacin as in the case of $\left[{ }^{3} \mathrm{H}\right]$ habekacin. The

Table 3. Relative uptake of $\left[{ }^{3} \mathrm{H}\right]$ dibekacin by $E$. coli cells in the presence or absence of habekacin.

\begin{tabular}{lcc}
\hline \multicolumn{1}{c}{ Addition } & $+2 \mu \mathrm{M}$ & $+10 \mu \mathrm{M}$ \\
\hline Dibekacin & 71 & 42 \\
Habekacin & 73 & 61 \\
[ ${ }^{3}$ H]Dibekacin & 138 & 228 \\
\hline
\end{tabular}

The number represents relative uptake of $\left[{ }^{3} \mathrm{H}\right]-$ dibekacin, and $100 \%$ uptake is $4.3 \times 10^{8}$ dibekacin molecules/cell. The exponentially growing cells of E. coli Q13 were incubated with $2 \mu \mathrm{M}\left[{ }^{3} \mathrm{H}\right]$ dibekacin at $37^{\circ} \mathrm{C}$ for 15 minutes in the presence or absence of habekacin, and transferred to ice bath.
Table 4. Relative binding of $\left[{ }^{3} \mathrm{H}\right]$ habekacin to $E$. coli ribosomes in the presence of various aminoglycoside antibiotics.

\begin{tabular}{lcc}
\hline \multicolumn{1}{c}{ Addition } & $+1 \mu \mathrm{M}$ & $+10 \mu \mathrm{M}$ \\
\hline Habekacin & 80 & 48 \\
Dibekacin & 82 & 67 \\
Amikacin & 86 & 75 \\
Kanamycin & 94 & 80 \\
Streptomycin & 90 & 81 \\
Neomycin & 68 & 46 \\
\hline
\end{tabular}

The number represents relative binding of $\left[{ }^{3} \mathrm{H}\right]$ habekacin to $E$. coli Q13 ribosomes, and $100 \%$ is the ratio of bound to free $\left[{ }^{3} \mathrm{H}\right]$ habekacin in the absence of antibiotics, which was 1.17. Equilibrium dialysis was carried out, using $1 \mu \mathrm{M}\left[{ }^{3} \mathrm{H}\right]-$ habekacin and $1 \mu \mathrm{M}$ ribosomes. 
uptake of $\left[{ }^{3} \mathrm{H}\right]$ dibekacin was reduced by habekacin (Table 3 ). The results suggest that these antibiotics possess a common transport system or binding site on the ribosome.

\section{Binding of $\left[{ }^{3} \mathrm{H}\right]$ Habekacin to E. coli Ribosomes in the Presence or}

\section{Absence of Various Aminoglycoside Antibiotics}

The effects of various aminoglycosides on the binding of $\left[{ }^{3} \mathrm{H}\right]$ habekacin to E. coli ribosomes were then studied, and the results are summarized in Table 4. The binding of habekacin was reduced by dibekacin, amikacin, kanamycin, streptomycin, or neomycin. The grade of inhibition by the aminoglycosides paralleled the one of cellular uptake (Table 2). The results support the assumption that the competition by the aminoglycosides for the bacterial uptake of habekacin occurs at the ribosomal level.

\section{Discussion}

The current experiments provide evidence that transport systems and ribosomal binding sites of habekacin are common to other aminoglycoside antibiotics. The kinetics of habekacin uptake (Fig. 2) are in accordance with the model of three sequential phases (EIP, EDP-I and EDP-II) typical for other aminoglycosides ${ }^{3)}$. The effect of habekacin is less than that of dibekacin on $\left[{ }^{3} \mathrm{H}\right]$ habekacin uptake (Fig. 4), suggesting a more (dibekacin) or less (amikacin) efficient competition for the same uptake channels and ribosomal binding sites. Competition by aminoglycosides for the cellular uptake of $\left[{ }^{3} \mathrm{H}\right]-$ habekacin may occur on the ribosome, because the degree of inhibition by the aminoglycosides of $\left[{ }^{3} \mathrm{H}\right]-$ habekacin binding to ribosomes was observed to parallel that of the cellular uptake.

From the viewpoint of cellular uptake and action on ribosomes, the present and previous ${ }^{7)}$ studies suggest that the antibacterial activity of habekacin is due to the dibekacin moiety of the molecule, and not to the 4-amino-2-hydroxybutyryl moiety.

\section{Acknowledgment}

The authors express their deep thanks to Dr. H. UMEZAwA, Institute of Microbial Chemistry, for his kind advice and cooperation in the present study.

\section{References}

1) Dubin, D. T.; R. Hancock \& B. D. Davis: The sequence of some effects of streptomycin in Escherichia coli. Biochim. Biophys. Acta 74: 476 489, 1963

2) BRyan, L. E. \& H. M. VAN DEN ElzEN: Gentamicin accumulation by sensitive strains of Escherichia coli and Pseudomonas aeruginosa. J. Antibiotics 28: 696 703, 1975

3) BRyan, L. E. \& H. M. VAN DEN Elzen: Streptomycin accumulation in susceptible and resistant strains of Escherichia coli and Pseudomonas aeruginosa. Antimicrob. Agents Chemother. 9: 928 938, 1976

4) Bryan, L. E.; T. Nicas, B. W. Holloway \& C. Crowther: Aminoglycoside-resistant mutation of Pseudomonas aeruginosa defective in cytochrome $\mathrm{C}_{552}$ and nitrate reductase. Antimicrob. Agents Chemother. 17: $71 \sim 79,1980$

5) Hurwitz, C.; C. B. Braun \& C. L. Rosano: Role of ribosome recycling and uptake of dihydrostreptomycin by sensitive and resistant Escherichia coli. Biochim. Biophys. Acta 652: 168 176, 1981

6) BRYAN, L. E. \& S. KWAN: Roles of ribosomal binding, membrane potential, and electron transport in bacterial uptake of streptomycin and gentamicin. Antimicrob. Agents Chemother. 23: 835 845, 1983

7) Tanaka, N.; K. Matsunaga, A. Hirata, Y. Matsuhisa \& T. Nishimura: Mechanism of action of habekacin, a novel amino acid-containing aminoglycoside antibiotic. Antimicrob. Agents Chemother. 24: $797 \sim 802,1983$

8) ChOI, E. C.; T. Nishimura \& N. TANAKA: Mutational alterations of either large or small ribosomal subunit for the kanamycin resistance. Biochem. Biophys. Res. Commun. 94: 755 762, 1980

9) Chor, E. C.; T. Nishimura, Y. TANaka \& N. TANAKa: In vivo and in vitro cross-resistance of kanamycinresistant mutants of $E$. coli to other aminoglycoside antibiotics. J. Antibiotics 33: 1527 1531, 1980 
10) Miyasaka, T.; D. Ikeda, S. Kondo \& H. Umezawa: Syntheses and properties of the 6"-deoxy or 4",6"dideoxy derivatives of the kanamycin antibiotics. J. Antibiotics 33: 527 532, 1980

11) Umezawa, H.; T. Miyasaka, H. Iwasawa, D. Ikeda \& S. Kondo: Chemical modification of 5,3',4'trideoxykanamycin B. J. Antibiotics 34: 1635 1640, 1981

12) Kondo, S.; K. Innuma, H. Yamamoto, K. Maeda \& H. UmeZawa: Syntheses of 1- $N$ - $\{(S)$-4-amino-2-hydroxybutyryl $\}$ kanamycin $\mathrm{B}$ and $-\mathbf{3}^{\prime}, 4^{\prime}$-dideoxykanamycin B active against kanamycin-resistant bacteria. J. Antibiotics 26: 412 415, 1973

13) Kondo, S.; K. Innuma, H. Yamamoto, K. Maeda \& H. Umezawa： Syntheses of (S)-4-amino-2-hydroxybutyryl derivatives of $3^{\prime}, 4^{\prime}$-dideoxykanamycin B and their antibacterial activities. J. Antibiotics 26: 705 707,1973

14) NAKAE, R. \& T. NAKAE: Diffusion of aminoglycoside antibiotics across the outer membrane of Escherichia coli. Antimicrob. Agents Chemother. 22: 554 559, 1982

15) Misumi, M.; T. Nishimura, T. Komai \& N. Tanaka: Interaction of kanamycin and related antibiotics with the large subunit of ribosomes and the inhibition of translocation. Biochem. Biophys. Res. Commun. $84: 358 \sim 365,1978$ 